\title{
Heterologous synthesis of the fungal alkaloid peramine
}

\author{
S.D. PIRLO, R.D. JOHNSON, C.R. VOISEY, A. KOULMAN and G.T. BRYAN \\ AgResearch, Grasslands, Private Bag 11008, Palmerston Nth, New Zealand
}

Endophytic fungi of temperate grasses form stable symbiotic associations that provide mutual benefits for both species. The plant hosts provide a niche environment for fungal growth, while the fungi synthesise secondary metabolites that protect the plant from biotic stresses such as insect and livestock foraging. The alkaloids produced by the fungal genera Epichloë and Neotyphodium are, for the most part, unique and include peramine, an apyrrolopyrazine that serves as a potent feeding deterrent of some insect pests of perennial ryegrass. The peramine molecule is composed of a lipophilic ring system and a hydrophilic guanidinium group (Fig. 1), both are structurally novel and not reported in any other insect feeding deterrent. Since no other species of fungi are known to produce peramine, this compound is restricted to endophytic infected grasses and therefore is currently limited as a means of controlling insect damage in agriculture. When endophytic fungi are cultured they produce only trace quantities of peramine. The heterologous synthesis of peramine in alternate fungal hosts and/or transgenic plants therefore has the potential to complement current agricultural insect management strategies.

Two fungal genes are believed to be necessary for the synthesis of peramine. The first is a non-ribosomal peptide synthetase (NRPS), an enzyme that is capable of conjugating amino acids to form short peptides, and the second is a phosphopantetheinyl transferase (PPTase), an enzyme that supplies the NRPS with an essential co-factor. The EF103 gene $(\sim 8,500 \mathrm{bp})$ has been proven to be required for peramine synthesis through targeted gene displacement experiments and encodes the predicted twomodule NRPS, PerA.

To demonstrate heterologous synthesis of peramine within another fungal species (which already contains a functional
PPTase gene) constitutive fungal expression vectors encoding perA (EF103) were synthesised using Gateway ${ }^{\circledR}$ cloning technology. Non-homologous recombination was performed to transform Penicillium paxilli with the expression cassettes, and mass spectrometry was used to rapidly detect peramine within the transgenic lines. The current peramine levels detected within the transgenic $P$. paxilli lines is low and further optimisation of culture conditions is required to increase synthesis. This is the first successful demonstration of the heterologous expression of a fungal NRPS in an alternate fungal species. The generation of transgenic Nicotiana tabaccum lines expressing both PerA and a PPTase is currently in progress.

Figure 1 The structure of peramine.

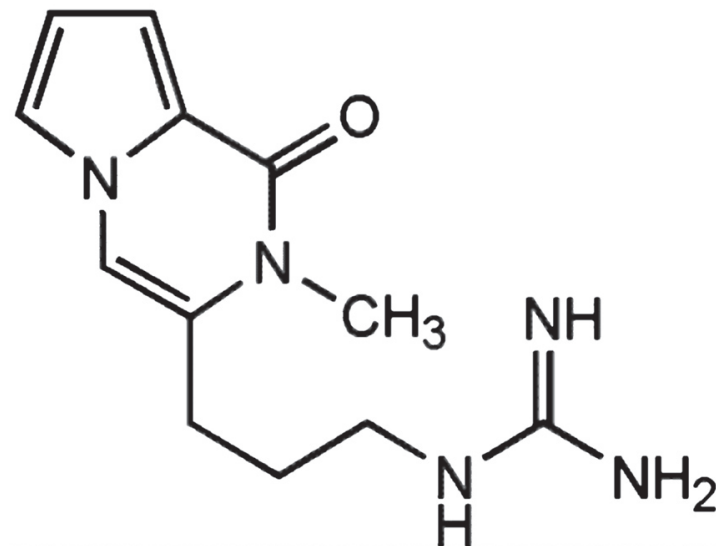

